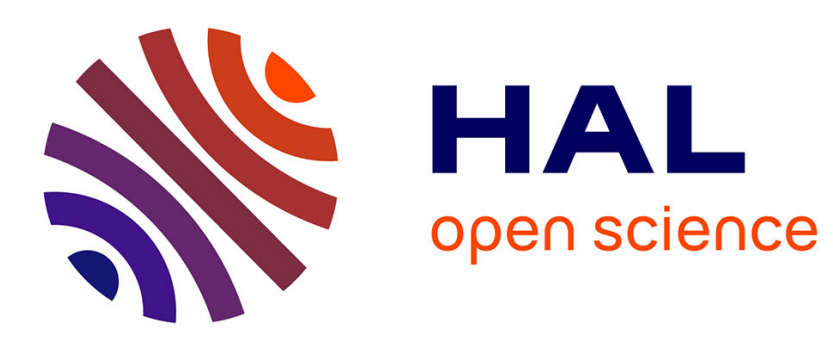

\title{
Closed loop optimal experiment design for on-line parameter estimation
}

Jun Qian, Madiha Nadri, Petru-Daniel Morosan, Pascal Dufour

\section{To cite this version:}

Jun Qian, Madiha Nadri, Petru-Daniel Morosan, Pascal Dufour. Closed loop optimal experiment design for on-line parameter estimation. IFAC-IEEE European Control Conference (ECC), Jun 2014, Strasbourg, France. pp.1813-1818. hal-01023213

\section{HAL Id: hal-01023213 https://hal.science/hal-01023213}

Submitted on 18 Jul 2014

HAL is a multi-disciplinary open access archive for the deposit and dissemination of scientific research documents, whether they are published or not. The documents may come from teaching and research institutions in France or abroad, or from public or private research centers.
L'archive ouverte pluridisciplinaire HAL, est destinée au dépôt et à la diffusion de documents scientifiques de niveau recherche, publiés ou non, émanant des établissements d'enseignement et de recherche français ou étrangers, des laboratoires publics ou privés. 
This document must be cited according to its final version which is published in a conference proceeding as:

\author{
J. Qian ${ }^{123}$, M. Nadri ${ }^{1}$, P.D. Morosan ${ }^{2}$, P. Dufour ${ }^{1}$ \\ 'Closed loop optimal experiment design for on-line nonlinear \\ system parameter identification", \\ IFAC-IEEE European Control Conference (ECC), June 24-27, \\ Strasbourg, France, pp. 1813-1818, 2014.
}

All open archive documents of Pascal Dufour are available at: http://hal.archives-ouvertes.fr/DUFOUR-PASCAL-C-3926-2008

The professional web page (Fr/En) of Pascal Dufour is: http://www.lagep.univ-lyon1.fr/signatures/dufour.pascal

The web page of this research group is: http://hal.archives-ouvertes.fr/SNLEP

1

Université de Lyon, Lyon, F-69003, France; Université Lyon 1;

CNRS UMR 5007 LAGEP (Laboratoire d'Automatique et de GEnie des Procédés),

43 bd du 11 novembre, 69100 Villeurbanne, France

Tel +33 (0) 472431845 - Fax +33 (0) 472431699

http://www-lagep.univ-lyon1.fr/ http://www.univ-lyon1.fr http://www.cnrs.fr

2

Acsysteme

Company Acsystème, 4 rue Ren é Dumont, 35000 Rennes, France.

Emails: \{jun.qian, petru-daniel.morosan\}@acsysteme.com

3 This $\mathrm{PhD}$ thesis is between the LAGEP and the french company Acsystème which is gratefully acknowledged for the funding. The french ministry of higher education and research, for the financial support of this CIFRE PhD thesis 2011/0876, is also acknowledged. 


\title{
Closed loop optimal experiment design for on-line parameter estimation
}

\author{
Jun QIAN ${ }^{1,2,3}$, Madiha NADRI ${ }^{1}$, Petru-Daniel MOROŞAN ${ }^{2}$ and Pascal DUFOUR ${ }^{1}$
}

\begin{abstract}
This paper focuses on the problem of closed loop on-line parameter identification for dynamic systems. An approach for the combined on-line optimal experiment design and model parameter identification is presented. Based on the observation theory and the model based predictive control theory, this approach aims to solve an optimal constrained control problem. During the designed experiment, the optimal time-varying input applied is computed at each current time to maximize the sensitivities of the model outputs with respect to the unknown model parameters which are also estimated online. The approach does not require to measure all the process state. Moreover constraints may be specified to maintain the system behavior in a prescribed region. A case study of chemical process is used to illustrate the developed approach.
\end{abstract}

\section{INTRODUCTION}

The increasing power of computer techniques leads to consider more complex and more realistic models for simulation, control or optimization of dynamic processes. Therefore all model parameters need to be numerically known. Many contributions and developments for parameter identification exist [1] and [2]. A classical technique for parameter estimation is the optimal experiment design (OED). However, many literature of OED is appealing to use past experimental data for offline model parameter identification, and separate parameter estimation from the optimal input design to get new experimental data [2] and [3]. Hence it increases the duration of use of the process for pure identification and the cost of these particular experiments (such as material fed at the process inlet, energy consumption during the experiment, output materials with undesired properties). Moreover, most applications of OED are reliable on linear or approximated linearized models [1] and [4], whereas in many areas such as biological and chemical processes, models are highly non linear. This paper focuses on the coupled on-line optimal experiment design and parameter estimation for multivariable systems, which are nonlinear in terms of state representation and/or in terms of parameters.

The commonly used criteria for OED aims to maximize the information contents in each experiment for parameter identification. Generally, the information contents are described as the Fisher Information Matrix (FIM) which is constituted by the sensitivities of the model output with

\footnotetext{
${ }^{1}$ Université de Lyon, F-69622, Lyon, France; Université Lyon 1, Villeurbanne; LAGEP, UMR 5007, CNRS, 43 bd du 11 novembre, 69100 Villeurbanne, France. Emails: \{qian, dufour, nadri\}@lagep.univ-lyon1.fr

${ }^{2}$ Company Acsystème, 4 rue René Dumont, 35000 Rennes, France. Emails: \{jun.qian, petru-daniel.morosan\}@acsysteme.com

${ }^{3}$ This $\mathrm{PhD}$ thesis is between the LAGEP and the french company Acsystème which is gratefully acknowledged for the funding. The french ministry of higher education and research, for the financial support of this CIFRE PhD thesis 2011/0876, is also acknowledged.
}

respect to the unknown parameters [2] and [3]. Recently, several authors combined on-line OED techniques with parameter estimation. In 2010, B. Jayasankar and his colleagues developed the OED for on-line parameter estimation in the multivariable case but without output constraint [5]. One year later, in [6], Y. Zhu and B. Huang used the steady state analysis to add linear equality constraints in an extended Kalman filter based approach to reduce the influence of poor initial conditions. These developed techniques for closed-loop identification purpose were addressed to open loop stable systems without input/output process constraint. Meanwhile, for real industrial applications, it is often necessary to account for process constraints (dealing with safety, maintain process state within bounds, production, economic performance, user comfort, etc). In such cases, closed loop constrained optimal control approach, using a Model Predictive Control (MPC) strategy, is a good choice for the controller.

MPC strategy is widely used since a few decades for industrial applications for regulation or trajectory tracking. For the OED, the main idea of a MPC approach is to determine an online optimal control sequence over a prediction horizon by maximizing the specified sensitivity criterion with the satisfaction of input and/or output constraints at each current moment. Therefore, the dynamic sensitivity model is established for feeding the sensitivity outputs into the controller. Receding-horizon design for parameter estimation has also been used in [13] for the linear case and in [6] for the nonlinear case. The new approach has been initially proposed in [8] where the authors considered first the monovariable case (a single input, a single measured state and a single unknown constant parameter) for stable nonlinear systems. Recently, we have showed that this approach may also be applied for an unstable nonlinear system with one input, two measured states and five unknown constant model parameters ([7]). The present paper extends this approach to another class of nonlinear systems.

In this approach, the observer design plays a very important role for online estimating the unknown model states (if any) but above all the unknown constant model parameters. Different observers have been proposed for nonlinear dynamic systems such as: high gain observer, extended Kalman filter (EKF) or adaptive-gain observer [9]. The choice of the observer type depends on the model structure and the observability property (which may depends on the sensitivity of the measurements with respect to the inputs). The convergence of observers is important for the parameter estimation. For example, in [6], the authors choose a classic EKF estimator for a continuous stirred tank reactor (CSTR) also studied in this paper. As we know, besides some numerical difficulties 
related to the gain calibration of the Riccati equation, it is not obvious to verify the mathematical convergence of this observer. Here, two high gain observers in cascade with proof of convergence are used.

This paper is organized as follows. In the section II, the proposed approach of closed-loop OED for on-line identification is presented. In section III, a practical application of this approach is given using a chemical process: a CSTR.

\section{PROPOSED CLOSED-LOOP OPTIMAL IDENTIFICATION APPROACH}

The proposed approach combines the closed-loop OED with an on-line identification for unknown model parameters of a nonlinear dynamic system base on ordinary differential equations. The non-linearity can be in terms of state representation and/or in terms of parameters. Based on the chosen model structure, an observer is designed for the augmented system resulting in a combined estimation of the unmeasured model states (if any) and the unknown model parameters. Then, a sensitivity model is stated to get the dynamic of all the sensitivities of the states and the output of the model with respect to the unknown model parameters. Finally, the OED problem is formulated by a cost function which maximizes the information content in the experiments. The constrained control problem is solved by a MPC strategy. The outputs of the process, the model, the observer, and the sensitivity model are used by the control law. The optimal inputs and the parameter estimations are determined on-line. Figure 1 presents the structure of this closed loop identification algorithm approach, where the principal components are described in the followings.

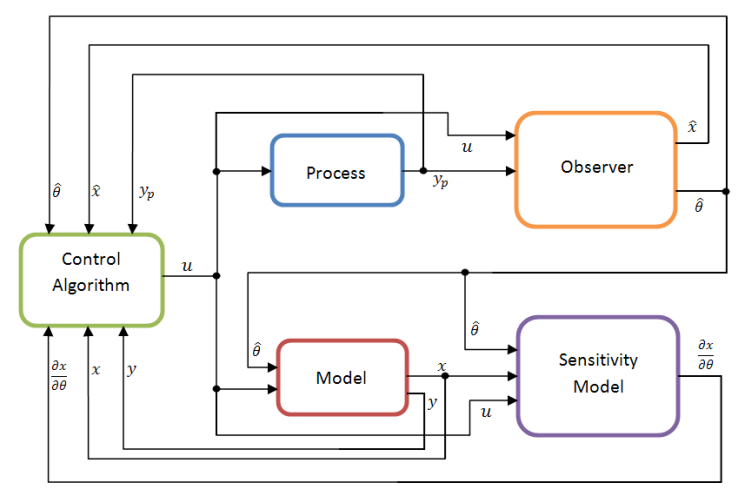

Fig. 1. Proposed closed loop control structure for on-line identification.

\section{A. Process}

The proposed approach can be used for processes that feature some dynamic behaviour. Meanwhile, at least one on-line output measure $y_{p}(t)$ must be available and at least one exogenous input $u(t)$ must be manipulable on-line by a controller. Some constraints may be specified on the magnitude and velocity of the manipulated inputs. Other constraints may be specified on the measured outputs or estimation of the process states. Hence, this covers a very large number of potential applications.

\section{B. Model}

The proposed approach can consider a large class of industrial processes which are modelled by a nonlinear dynamic multivariable model based on ordinary differential equations:

$$
(M)\left\{\begin{array}{l}
\dot{x}(t)=f(x(t), \theta, u(t)) \\
y(t)=h(x(t), \theta, u(t)),
\end{array}\right.
$$

where $x \in \mathcal{R}^{n}$ is the state vector, $y \in \mathcal{R}^{p}$ is the vector of measured outputs, $u \in \mathcal{U} \subset \mathcal{R}^{m}$ denotes the vector of known manipulated inputs, $\theta \in \mathcal{R}^{q}$ is the unknown model parameters vector, $f$ and $h$ are nonlinear functions of suitable dimensions.

Assumption 1: In this study, the unknown model parameters are all constant.

Assumption 2: In the system (1), $f$ and $h$ are $C^{\infty}$ w.r.t. their arguments.

\section{Observer design}

An observer can be constructed to give an estimation $\hat{x}(t)$ for the state $x(t)$ by using a judicious model state of the process and the knowledge of $u\left(t^{\prime}\right)$ and $y\left(t^{\prime}\right)$ for $0 \leq$ $t^{\prime} \leq t$. Moreover, in terms of model parameter identification, the observer has to be designed to estimate on-line not only model states (if any), but also the unknown constant parameter vector $\theta$. Hence, the observer design is based on the following augmented model :

$$
\left(M_{a}\right)\left\{\begin{array}{l}
\dot{x}(t)=f(x(t), \theta, u(t)) \\
\dot{\theta}=0 \\
y(t)=h(x(t), \theta, u(t))
\end{array}\right.
$$

In the follows, we denote the augmented state vector by $x_{a}=$ $\left[\begin{array}{ll}x & \theta\end{array}\right]^{T}$ and the vector function $f_{a}=\left[\begin{array}{ll}f & 0\end{array}\right]^{T}$.

Definition 1: A global observer for system (2) can be given by a dynamical system under the form:

$$
(O) \begin{cases}\dot{\hat{x}}_{a}(t) & =f_{a}\left(\hat{x}_{a}(t), u(t)\right)+\ldots \\ & \ldots g_{a}\left(t, h\left(\hat{x}_{a}(t), u(t)\right)-y_{p}(t)\right) \\ \text { with: } & g_{a}(t, 0)=0\end{cases}
$$

such that

i) if $\hat{x}_{a}(0)=x_{a}(0)$, then $\hat{x}_{a}(t)=x_{a}(t), \quad \forall t \geq 0$;

ii) if $\forall x_{a}(0), \forall \hat{x}_{a}(0)$, then $\lim _{t \rightarrow+\infty}\left\|\hat{x}_{a}(t)-x_{a}(t)\right\|=0$, where $g_{a}$ is a function of the output estimation error to be designed and $y_{p}$ is the process output vector (real measures).

Our estimation problem consists in determining a gain $g_{a}$ such that the estimation error $e(t)=x_{a}(t)-\hat{x}_{a}(t)$ converges asymptotically to zero.

Remark 1: In general, it is not required to measure all the model states $x(t)$ for our proposed approach. But it is necessary and sufficient to measure a combination of some states which allow to design an observer for the estimation of the unknown states and unknown parameters, and also to study the sensitivity of the model with respect to the unknown parameters. In order to better illustrate the different steps of the implementation of the approach, we can now assume that the whole state is measured. 


\section{Sensitivity model}

The sensitivity analysis tells us how the unknown parameters $\theta$ affect the system state $x$. Thus we define the sensitivity function as:

$$
\frac{\partial}{\partial t} x_{\theta}=\frac{d}{\partial t}\left(\frac{\partial x}{\partial \theta}\right)=\frac{\partial}{\partial \theta}\left(\frac{\partial x}{\partial t}\right)=\frac{\partial f}{\partial \theta}+\frac{\partial f}{\partial x} \frac{\partial x}{\partial \theta} .
$$

Based on this definition, the sensitivity model is:

$$
\left(M_{\theta}\right)\left\{\begin{array}{l}
\dot{x}_{\theta}(t)=\frac{\partial f(x(t), \theta, u(t))}{\partial x} x_{\theta}+\frac{\partial f(x(t), \theta, u(t))}{\partial \theta} \\
y_{\theta}(t)=x_{\theta}(t),
\end{array}\right.
$$

where $x_{\theta} \in R^{n \times q}$ and $y_{\theta} \in R^{n \times q}$ are the sensitivity matrix of the states (the outputs) with respect to the unknown model parameters. Since physical values involved in the sensitivities have usually different scales and units, each sensitivity is normalized with the relative-sensitivity function:

$$
\begin{cases}\bar{x}_{\theta}(i, j)=\frac{\theta_{j}}{x_{i}} x_{\theta}(i, j) ; \quad i=1, \cdots, n ; j=1, \cdots, q \\ \bar{y}_{\theta}(i, j)=\frac{\theta_{j}}{y_{i}} y_{\theta}(i, j) ; \quad i=1, \cdots, n ; j=1, \cdots, q\end{cases}
$$

\section{E. MPC strategy}

MPC is widely used in the industry, particularly for solving an optimal control problem (trajectory tracking, processing time minimization, etc) with input constraints or/and with output constraints:

$$
\left\{\begin{array}{l}
\text { input constraints, }(\forall k>0): \\
\left\{\begin{array}{l}
u_{\min } \leqslant u(l) \leqslant u_{\max } \\
\Delta u_{\min } \leqslant \frac{u(l)-u(l-1)}{T_{s}} \leqslant \Delta u_{\max }
\end{array}\right. \\
\text { state/output constraints, }(\forall k>0): \\
c_{n}\left(y_{p}(k), \hat{x}_{a}(k), y(l), x(l), u(l)\right) \leqslant 0, \\
\forall n \in I=1, \ldots N_{\text {soc }}
\end{array}\right.
$$

where $N_{s o c}$ is the number of the state/output constraints. The objective of the online optimal control problem is here to maximize the information content of the experiment, namely to maximize the sensitivities of the model states with respect to the unknown constant parameters at each instant. To do so, we use the FIM based on the sensitivity matrix $\bar{Z}_{k}$ at the current instant $\mathrm{k}^{1}$, composed of the normalized outputs sensitivity $\bar{y}_{\theta}$ :

$$
\bar{Z}_{k}=\left[\begin{array}{lcll}
\left.\bar{y}_{\theta}(1,1)\right|_{k} & \left.\bar{y}_{\theta}(1,2)\right|_{k} & \ldots & \left.\bar{y}_{\theta}(1, q)\right|_{k} \\
\left.\bar{y}_{\theta}(2,1)\right|_{k} & \ddots & & \vdots \\
\vdots & & \ddots & \vdots \\
\left.\bar{y}_{\theta}(n, 1)\right|_{k} & \ldots & \ldots & \bar{y}_{\theta}(n, q)_{k}
\end{array}\right] .
$$

\footnotetext{
${ }^{1}$ To simplify the notation, here, $s(k)$ (resp. $s(l)$ ) represents the value of the signal $s$ at the current (resp. future) discrete time $k \times T_{s}$ (resp. $\left.l \times T_{s}\right)$, where $T_{s}$ is the constant sampling time. For the input, a zero order hold is used between two consecutive sampling times. The various models are still formulated in a continuous framework and are solved numerically. Hence, sampled values may be taken at any discrete time. The initial value of the model state at $k$ is obtained from the real measurements or from the state estimated by the observer. It is assumed that process data may also be sampled at the same rate.
}

Then, the FIM $M_{k}$ is given by:

$$
M_{k}=\bar{Z}_{k}^{T} \bar{Z}_{k}
$$

In order to optimize the information contents over a receding horizon, we consider the cost function $J$ :

$$
\left\{\begin{aligned}
J= & \phi\left(F\left(\left.\bar{y}_{\theta}\right|_{l \mid k}, u_{l \mid k}, y_{p}(k), \hat{x}_{a}(k), x(k), y(k)\right)\right), \\
\text { with } & F\left(\left.\bar{y}_{\theta}\right|_{l \mid k}, u_{l \mid k}, y_{p}(k), \hat{x}_{a}(k), x(k), y(k)\right) \\
& =\frac{1}{N_{p}} \sum_{l=k+1}^{k+N_{p}} M_{l \mid k}
\end{aligned}\right.
$$

where $\phi($.$) is the criteria described below and F$ is a matrix which represents the average information during a predictive horizon $N_{p}$, at the current discrete time $k . M_{l \mid k}$ is the FIM computed over the prediction horizon $N_{p}$ at the current time k. Finally, the E-optimality criterion is defined to maximize the smallest eigenvalue of the matrix $F$. Geometrically, it minimizes the size of the major axis of the join confidence region of the estimated parameters. This allows to choose the optimal input $u^{\star}(k)$ to apply at $k$ :

$$
\left\{\begin{array}{l}
u_{l \mid k}^{*}=\arg \max _{u_{l \mid k}}\left(J\left(u_{l \mid k}\right)=\frac{\lambda_{\min }(F)}{\lambda_{\max }(F)}\right) \\
u_{l \mid k}=\left\{u(k) \ldots u(l) \ldots u\left(k+N_{p}\right)\right\}, l \in\left[k k+N_{p}\right] .
\end{array}\right.
$$

This procedure is repeated at the next sampling time. Finally, the optimal inputs and the parameter estimations are both determined online.

\section{CASE STUdy: CSTR}

The proposed approach is now detailed step by step on a simulation case from chemical engineering.

\section{A. Process}

In this section, the CSTR discussed in [6] for an identification problem is used: it is a first-order exothermic irreversible reaction. In [6], a steady state analysis led to create linear equality constraints, whereas no other real constraint were specified.

\section{B. Model}

This nonlinear dynamic system is described as follows:

$$
\begin{cases}\dot{c_{A}}(t)= & \frac{q}{V}\left(c_{A}^{f}-c_{A}(t)\right)-k_{0} e^{-\frac{E}{R T(t)}} c_{A}(t) \\ \dot{T}(t)= & \frac{q}{V}\left(T_{f}-T(t)\right)+\frac{\Delta H}{\rho C_{p}} k_{0} e^{-\frac{E}{R T(t)}} c_{A}(t) \\ & +\frac{U A}{\rho V C_{p}}\left(T_{c}(t)-T(t)\right)\end{cases}
$$

where the temperature of cooling jacket $T_{c}(t)$ is the manipulated input. The concentration of component $\mathrm{A}, c_{A}(t)$, and the reactor temperature, $T(t)$, are the two states and the two outputs. The parameters vector is $\theta=\left[\begin{array}{ll}\frac{1}{\rho} & k_{0}\end{array}\right]$. The descriptions and values of the known (resp. target for unknown) parameters in the system are listed in the table I (resp. II) . 
TABLE I

CSTR: KNOWN MODEL PARAMETERS.

\begin{tabular}{lcc}
\hline Parameter (units) & Symbol & Value \\
\hline Volumetric flowrate $\left(\mathrm{m}^{3} / \mathrm{s}\right)$ & $q$ & 100 \\
Volume of CSTR $\left(\mathrm{m}^{3}\right)$ & $V$ & 100 \\
Heat capacity of A-B mixture $(\mathrm{J} / \mathrm{kg} K)$ & $C_{p}$ & 0.239 \\
Heat of reaction for A-B $(\mathrm{J} / \mathrm{mol})$ & $\Delta H$ & $5 \times 10^{4}$ \\
$-(W / K)$ & $\mathrm{UA}$ & $5 \times 10^{4}$ \\
$U:$ overall heat transfer coefficient & & \\
$\left(W / m^{2} K\right)$ & & \\
$A:$ Area, this value is specific for the & & \\
$\mathrm{U}$ calculation $\left(\mathrm{m}^{2}\right)$ & & \\
Feed concentration $\left(m o l / m^{3}\right)$ & $c_{a}^{f}$ & 350 \\
Feed temperature $(K)$ & $T^{f}$ & \\
Exponential factor $(K)$ & $\frac{E}{R}$ & 8750 \\
$E:$ Activation energy in the Arrhenius & & \\
equation $(J / m o l)$ & \\
$R:$ Universal gas constant, 8.31451 & \\
$(J / m o l / K)$ & \\
\hline
\end{tabular}

TABLE II

CSTR: TARGET FOR THE UNKNOWN MODEL PARAMETERS

\begin{tabular}{lcc}
\hline Parameter (units) & Symbol & Value \\
\hline Density of A-B mixture $\left(\mathrm{kg} / \mathrm{m}^{3}\right)$ & $\rho$ & 1000 \\
Pre-exponential factor $\left(\mathrm{s}^{-1}\right)$ & $k_{0}$ & $7.2 \times 10^{10}$ \\
\hline
\end{tabular}

\section{Design of two observers in cascade}

Using the notations: $\alpha=\left[\frac{q}{v} ; c_{A}^{f} ;-\frac{E}{R} ; T_{f} ; \frac{\Delta H}{C_{p}} ; \frac{U A}{V C_{p}}\right]$ (the known constant model parameters) and $x_{a}(t)=$ $\left[c_{A} ; T ; \frac{1}{\rho} ; k_{0}\right]$ (the augmented state vector), the augmented system is ${ }^{2}$ :

$$
\left\{\begin{aligned}
\dot{x}_{a 1}(t)= & \alpha_{1}\left(\alpha_{2}-x_{a 1}(t)\right)-x_{a 4} e^{\frac{\alpha_{3}}{x_{a 2}(t)}} x_{a 1}(t) \\
\dot{x}_{a 2}(t)= & \alpha_{1}\left(\alpha_{4}-x_{a 2}(t)\right)+\alpha_{5} x_{a 3} x_{a 4} e^{\frac{\alpha_{3}}{x_{a 2}(t)}} x_{a 1}(t) \\
& +\alpha_{6} x_{a 3}\left(u(t)-x_{a 2}(t)\right) \\
\dot{x}_{a 3}(t)= & 0 \\
\dot{x}_{a 4}(t)= & 0 \\
y(t)= & {\left[\begin{array}{l}
x_{a 1}(t) \\
x_{a 2}(t)
\end{array}\right] . }
\end{aligned}\right.
$$

In order to estimate the unknown parameters, the augmented model (13) can be rewritten as two interconnected subsystems. To do so, we introduce the following notations $: z^{1}=\left[\begin{array}{cc}x_{a 1} & x_{a 4}\end{array}\right]^{T}, z^{2}=\left[\begin{array}{cc}x_{a 2} & x_{a 3}\end{array}\right]^{T} ;$
$A_{1}(y)=\left[\begin{array}{cc}0 & -y_{1} \exp \left(\frac{\alpha_{3}}{y_{2}}\right) \\ 0 & 0\end{array}\right] ; \varphi_{1}(y)=\left[\begin{array}{c}\alpha_{1} \alpha_{2}-\alpha_{1} y_{1} \\ 0\end{array}\right] ;$
$A_{2}\left(u, y, \eta_{1}\right)=\left[\begin{array}{cc}0 & \alpha_{5} z_{2}^{1} \exp \left(\frac{\alpha_{3}}{y_{2}}\right) z_{1}^{1}+ \\ 0 & \alpha_{6}\left(u-y_{2}\right)\end{array}\right] ;$
$\varphi_{2}(y)=\left[\begin{array}{c}\alpha_{1} \alpha_{4}-\alpha_{1} y_{2} \\ 0\end{array}\right] ; C_{1}=C_{2}=\left[\begin{array}{ll}1 & 0\end{array}\right]$.

Using these notations, we get the following typical cascade affine system up to output injection [10]

\footnotetext{
${ }^{2}$ For simplicity, we use the notation $(\cdot)_{i}=(\cdot)(i)$
}

$$
\left\{\begin{array}{l}
\dot{z}^{1}=A_{1}(y) z^{1}+\varphi_{1}(y) \\
\dot{z}^{2}=A_{2}\left(u, y, z^{1}\right) z^{2}+\varphi_{2}(y) \\
y=\left[\begin{array}{l}
C_{1} z^{1} \\
C_{2} z^{2}
\end{array}\right] .
\end{array}\right.
$$

Assuming that for each single output subsystem $i$, one can design an observer $\left(\mathcal{O}_{i}\right)(i=1,2)$, the point is to design an observer for the whole system from the interconnection of two observers $\left.\left(\mathcal{O}_{1}\left(\hat{z}^{1}\right)\right)\right)$ and $\left(\mathcal{O}_{2}\left(\hat{z}^{1}, \hat{z}^{2}\right)\right)$.

Based on these two fully interconnected subsystems, one use two classical observers as follows :

- Subsystem in $z^{1}$ is a particular case of the form treated in [14], then using the same assumptions, the following high gain observer can be designed

$$
\dot{\hat{z}}^{1}=A_{1}(y) \hat{z}^{1}+\varphi_{1}(y)-G\left(C_{1} \hat{z}^{1}-y_{1}\right),
$$

where $\hat{z}^{1}$ is the estimated state vector, $G=\Delta^{-1} S_{\lambda}^{-1} C_{1}^{T}$, $\Delta=\left[\begin{array}{cc}1 & 0 \\ 0 & A_{1}(1,2)\end{array}\right], S_{\lambda}=\left[\begin{array}{cc}\frac{1}{\lambda_{1}} & -\frac{1}{\lambda^{2}} \\ -\frac{1}{\lambda^{2}} & \frac{2}{\lambda^{3}}\end{array}\right]$ and $\lambda$ the observer tuning parameter.

- Subsystem in $z^{2}$ is state-affine system with output injection for which an high gain observer can be designed (for more details see [9]). To do so, we introduce the following assumption.

Assumption 3: Let $\Phi_{1}(t ; s)$ be the transition matrix of the state affine subsystem in $z^{2}$ which satisfy

$$
\begin{aligned}
& \frac{\partial \Phi_{u}}{\partial t}\left(t ; z^{1}\right)=A_{2}\left(u, y, z^{1}(t)\right) \Phi_{u}\left(t ; z^{1}\right), t \in \mathcal{R} \\
& \text { with } \Phi_{u}\left(0 ; z^{1}\right)=I .
\end{aligned}
$$

For every $z^{1}(\cdot)$, solution for the subsystem in $z^{1}$, there exists two positive constants $T$ and $\eta$ such that

$$
\int_{t-T}^{t} \Phi_{u}\left(s ; z^{1}\right)^{\prime} C_{2}^{\prime} C_{2} \Phi_{u}\left(s ; z^{1}\right) \mathrm{d} s \geq \eta I, \forall t \in \mathcal{R} .
$$

Under this assumption, an observer can be given as

$$
\left\{\begin{array}{l}
\dot{\hat{z}}^{2}=A_{2}\left(u, y, \hat{z}^{1}\right) \hat{z}^{2}+\varphi_{2}(y)-S^{-1} C_{2}^{T} R\left(C_{2} \hat{z}^{2}-y_{2}\right) \\
\dot{S}=-\mu S-A_{2}^{T}\left(u, y, \hat{z}^{1}\right) S-S A_{2}^{T}\left(u, y, \hat{z}^{1}\right)+C_{2}^{T} R C_{2}
\end{array}\right.
$$

where $\hat{z}^{2}$ is the estimated state vector, $S$ is a symmetric positive definite (S.P.D.) matrix $2 \times 2, \mathrm{R}$ is a positive constant, and $\mu$ is the tuning parameter for the observer.

Using the above, we can now state our result,

Theorem 1: there exist two constants $\mu^{*}$ and $\lambda^{*}$ such that for every $\mu \geq \mu^{*}$ and $\lambda \geq \lambda^{*}$, the following system is an asymptotic observer for the whole system (14)

$$
\left\{\begin{array}{l}
\dot{\hat{z}}^{1}=A_{1}(y) \hat{z}^{1}+\varphi_{1}(u, y)-G\left(C_{1} \hat{z}^{1}-y_{1}\right) \\
\dot{\hat{z}}^{2}=A_{2}\left(u, y, \hat{z}^{1}\right) \hat{\eta}_{2}+\varphi_{2}(y)-S^{-1} C_{2}^{T} R\left(C_{2} \hat{z}^{2}-y_{2}\right) \\
\dot{S}=-\mu S-A_{2}^{T} S-S A_{2}+C_{2}^{T} R C_{2}
\end{array}\right.
$$

where $\hat{z}^{i}$ is the estimated state vector, $G$ and $\lambda$ are given by (15) and $\mu$ is given by (17).

Sketch of proof 1: Let us first consider the subsystem in $z^{1}$. Using the results given in [14] and [9], and the same assumptions therein, we have directly the estimation error $e_{1}(t)=z^{1}(t)-\hat{z}^{1}(t)$ that converges asymptotically towards zero. To complete the proof, we have to study the behaviour 
of the second part of the estimation error $e_{2}(t)=z^{2}-\hat{z}^{2}$, which depends on the convergence of $e_{1}$. In [12], the authors gave the proof of the observer convergence for the same class of affine systems with output injection but without interconnection (there was no dependence on $z^{1}$ ). To use of this result, it suffices to show that :

if $S$ is solution of

$$
\dot{S}=-\theta S-S A_{2}\left(\hat{z}^{1}\right)-A_{2}\left(\hat{z}^{1}\right)^{T} S+C_{2}^{T} C_{2},
$$

then there exist two positive constants $a_{1}, a_{2}$ such that

$$
a_{1} I \leq S(t) \leq a_{2} I
$$

For more details, see [12].

Based on (19) and using classical Lyapunov approach, it can be shown: $\lim _{t \rightarrow+\infty} e_{2}(t)=0$

\section{Sensitivity model}

The definition (4)-(5) of the sensitivity model in section II is used to get the sensitivity model $\left(x_{i \theta j}=\frac{\partial x_{i}}{\partial \theta_{j}}\right)$. These sensitivities are the normalized by (6).

\section{E. MPC strategy}

The input constraints in this case study are defined by:

$$
250 K \leqslant T_{c}(l) \leqslant 320 K, \forall l \in\left[k, k+N_{p}\right]
$$

and an output constraint may be present or not in closed loop:

$$
c_{A}(l) \leqslant 0.95 \mathrm{~mol} / \mathrm{m}^{3}, \forall l \in\left[k, k+N_{p}\right] .
$$

\section{F. Results and discussion}

The MPC strategy solves the optimization problem at the current time $k$ to compute the optimal temperature of cooling jacket $T_{c}$. At the same time, the constant unknown parameters $1 / \rho$ and $k_{0}$ are estimated by the observers.

This proposed optimal identification approach is programmed in the ODOE4OPE ${ }^{3}$ software which is used and developed in our research work. In order to evaluate this approach, three different cases are considered:

- Case 1: identification in open loop with a constant input $\left(T_{c}=300 \mathrm{~K}\right)$ satisfying the input constraints;

- Case 2: identification in closed loop by using the proposed approach with the input constraints (20);

- Case 3: identification in closed loop by using the proposed approach with the input constraints (20) and the output constraint (21).

These three cases are all studied under the same initial conditions and using the same parameter values which are defined in table III.

In case 1, a constant input value is applied which allows that both estimated model parameters converge to their target value (Fig. 2 and Fig. 3). In closed loop, (case 2) both estimated model parameters converge to their target faster than in the open loop case (between 30 and 50\% faster) and with less error before the final convergence, especially for

\footnotetext{
${ }^{3}$ ODOE4OPE: optimal design of experiments for on-line parameter estimation: http://ODOE4OPE.univ-lyon1.fr
}

TABLE III

INITIAL CONDITIONS AND PARAMETER VALUES FOR THE SIMULATIONS.

\begin{tabular}{lcc}
\hline Initial conditions & Symbol & Value $(\mathrm{uSI})$ \\
\hline Initial parameter values & {$\left[\hat{\theta}_{1}(0) \hat{\theta}_{2}(0)\right]$} & {$\left[\frac{1}{1100} 6.3 \times 10^{10}\right]$} \\
Initial model states & {$\left[x_{1}(0) x_{2}(0)\right]_{m}$} & {$[0.9305]$} \\
Initial estimated states & {$\left[\hat{x}_{1}(0) \hat{x}_{2}(0)\right]$} & {$\left[\begin{array}{ll}0.9 & 305\end{array}\right]$} \\
Tuning parameters & {$[\lambda \mu]$} & {$\left[\begin{array}{ll}1 & 1\end{array}\right]$} \\
S.P.D matrix in (17) & $S_{l}(0)$ & {$\left[\begin{array}{cc}1 & 0 \\
0 & 1\end{array}\right]$} \\
Weight matrix in (17) & $R$ & 3 \\
Time of the simulation & $T_{\text {end }}(s)$ & 50 \\
Sampling period & $T_{s}(s)$ & 0.5 \\
Prediction horizon & $N_{p}$ & 10 \\
\hline
\end{tabular}

$\rho$. Indeed, the controller is able to find better control actions (in the sense of the criteria Fig. 4 and Fig. 5) which leads to have a time-varying input profile (Fig. 6) which increases from the initial point $u(0)=270 \mathrm{~K}$ and converge to $282.7 \mathrm{~K}$. The result is that $c_{A}$ increases monotonously and stops over $0.972 \mathrm{~mol} / \mathrm{m}^{3}$ (Fig. 7). A smaller value is used to define the upper bound in the new output constraint (21) which is always satisfied (Fig. 7) in the case 3: here, both estimated model parameters converge to their target value, but slower than in case 2 (but still quicker than in case 1). The input is still time-varying and it is found to be between the two previous solutions (Fig. 6).

Therefore, the approach is able to decrease the operating time needed to estimate the CSTR model parameters (hence decrease the cost of the experiment) while keeping the state inside a prescribed constrained set, which was not the case with the method employed in [6].

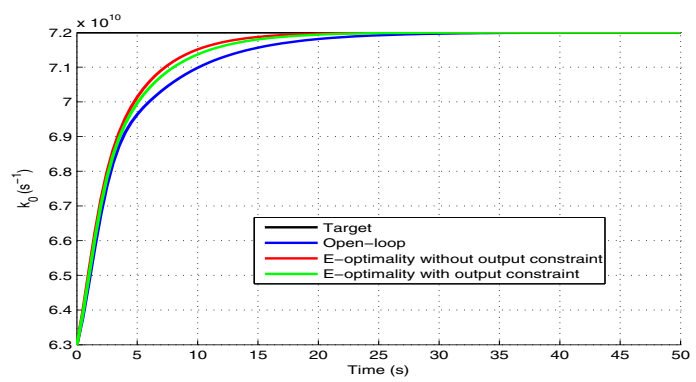

Fig. 2. Parameter estimation: $k_{0}$.

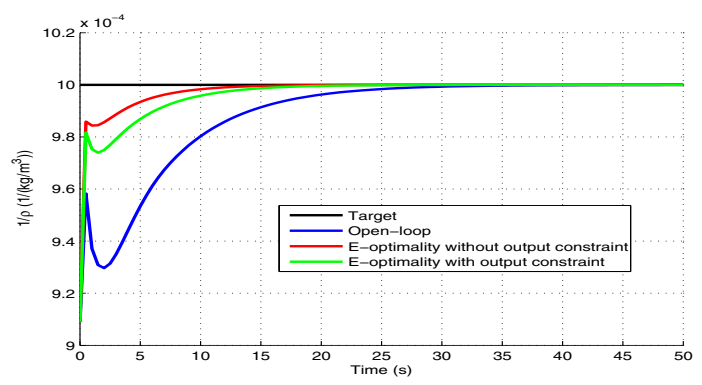

Fig. 3. Parameter estimation: $1 / \rho$. 


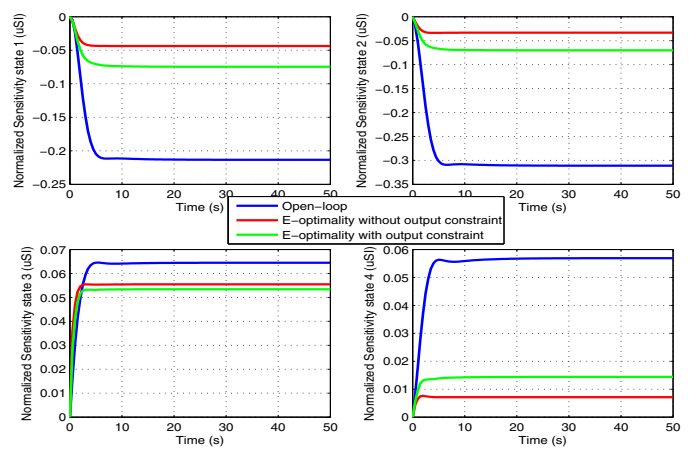

Fig. 4. Normalized sensitivities of model states with respect to the estimated parameters.

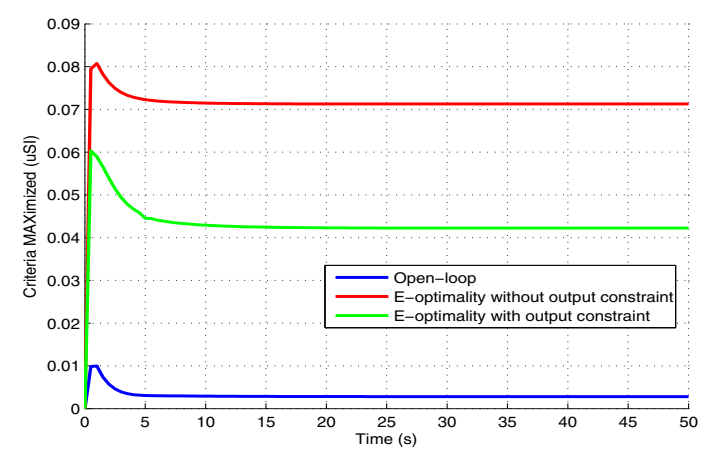

Fig. 5. Criterion E maximized.

\section{CONCLUSION}

In this paper, an approach combining the closed loop online parameter identification with optimal design of experiments based on observer techniques has been proposed. It aims to compute online the optimal profile of input to maximize the sensitivities of process outputs with respect to the unknown constant model parameters which are estimated at the same time. Using nonlinear observer techniques coupled to a model based predictive controller, a cost function based on the sensitivity criterion has been optimized. The efficiency of the proposed algorithm has been evaluated through a classical chemical process. The simulation results show that the closed loop optimal identification approach can find the optimal input and give a faster parameter estimation than the experiment in the open loop, while the constraints specified on the inputs and outputs can be well satisfied.

\section{REFERENCES}

[1] T.S. Ng, G.C. Goodwin, T. Söderström, Optimal experiment design for linear systems with input-output constraints, Automatica, Vol. 13, Issue 6, 1977, Pages 571-577.

[2] L. Ljung, System identification: Theory for the user, Prentice Hall, 1999.

[3] E. Walter and L. Pronzato, Identification of parametric models from experimental data, Springer-Verlag, 1994.

[4] L. Keviczky, Design of experiments for the identification of linear dynamic systems, Technometrics, 1975, Vol.17, No. 3, pp. 303-308.

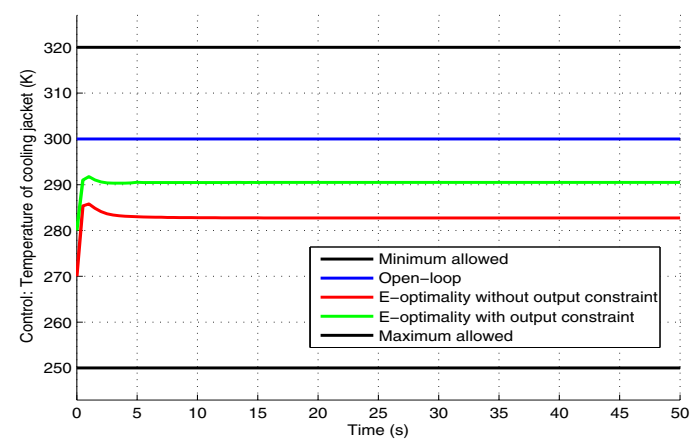

Fig. 6. Input: temperature of cooling jacket $T c$.

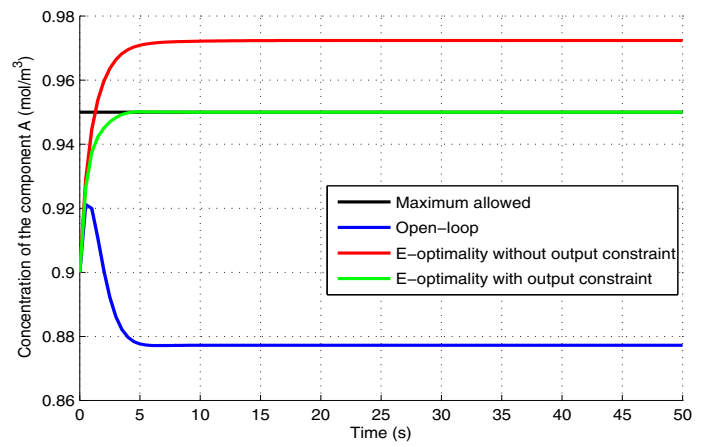

Fig. 7. Output : concentration of component $\mathrm{A} c_{A}$.

[5] B. Jayasankar, B. Huang and A. Ben-Zv, Receding horizon experiment design with application in sofc parameter estimation, in Proceedings of the International Symposium on Dynamics and Control of Process Systems, 2010, pp.527-532, Leuven, Belgium.

[6] Y. Zhu and B. Huang, Constrained receding-horizon experiment design and parameter estimation in the presence of poor initial conditions, AIChE Journal, October 2011, Vol. 57, No. 10, pp. 2808-2820.

[7] J. Qian, P. Dufour and M. Nadri, Observer and model predictive control for on-line parameter identification in nonlinear systems, in Proceeding of the 10th International Symposium on Dynamics and Control of Process Systems DYCOPS, Mumbai, India, 2013, pp. 571576.

[8] S. Flila, P. Dufour and H. Hammouri, Optimal input design for online identification: a coupled observer-MPC approach, in Proceedings of the 17th IFAC World Congress 2008, Seoul, South Korea, July 6-11, 2008, Paper 1722, pp. 11457-11462.

[9] G. Besançon, nonlinear observers and applications, Lecture Notes in Control and Information Sciences 363, Springer-Verlag Berlin Heidelberg, 2007.

[10] G. Besançon, G.Bornand and H. Hammouri. Observer Synthesis for a class of Nonlinear Control Systems, European Journal of Control, 1996, Vol.2, No.3, pp.176-192.

[11] N. Boizot, E. Busvelle and J.-P. Gauthier, An adaptive high-gain observer for nonlinear systems, Automatic, Vol. 46, Issue 9, 2010, pp. 1483-1488.

[12] G. Bornard, N. Couenne, F. Celle, Regularly persistent observers for bilinear systems, Proceedings of the 29th International Conference on Nonlinear Systems New Trends in Nonlinear System Theory, Vol. 122Springer-Verlag (1988)

[13] P. Vuthandam and M. Nikolaou, Constrained MPCI: A weak persistent excitation approach, AIChE Journal, 1997, Vol. 43, No. 9, pp. 22792288.

[14] M. Farza, M. Nadri and H. Hammouri, Nonlinear observation of specific growth rate in aerobic fermentation processes, Bioprocess and Biosystems Engineering, 2000, Vol. 23, No.4, pp. 359-366. 